\title{
Counterterms in Supergravity
}

\author{
Bern et al, \\ Z. Bern, D. Dunbar, L. Dixon, B. Julia, M. Perelstien, J. Rozowsky, D. Seminara \\ and M. Trigante \\ Talk presented by D. Dunbar \\ University of Wales Swansea \\ E-mail: 'id.c.dunbar@swan.ac.uki
}

ABstract: We examine the ultraviolet behaviour of supergravity theories as a function of dimension and number of supercharges. We do so by the computation of one and two-loop physical on-shell four point amplitudes. For maximal supergravity, our computations prove the non-renomalisability of supergravity for $D \geq 6$ (including the maximal $D=11$ case) and give strong evidence for the existance of a five-loop counterterm in $D=4$. For type I supergravity our results indicate similar patterns. We shall also explore a remarkable relationship between gravity amplitudes and those of Yang-Mills theories. In many ways gravity calculations discover features which relate to the equivalent Yang-Mills features by a squaring proceedure.

\section{KEYwORDS: 'Ŝpergravity Extended Supersymmetry Superstrings Quantum Gravitỳ.'}

\section{Motivation}

Supergravity is one of the key theories in understanding quantum gravity. In itself, it is almost certainly not a renomalisable theory so must appear as the low energy limit of another theory such as M-theory. However, supergravity and variations thereof will be the effective theories which describe quantum gravity at energies less than the (colossally large) Planck scale.

We shall study the ultra-violet behavior of supergravity theories for two reasons: firstly we wish to prove the conjectured bad behavior of these theories: secondly we hope to understand some features of the physics at the Planck scale. Adding counterterms is a well defined, but unpredictive at the Planck scale, way to regulate a theory. A physical regulator should in some sense provide the same regulation but within a predictive context. The symmetries and structure of the physical theory might well find themselves mirrored in the counterterm structure.

One of the themes of this TMR network has been the use of integrability in understanding

\footnotetext{
*Research supported by TMR contract FMRX-CT920012
}

two dimensional field theories. Unfortunately the enormous success in two dimensions has not, yet, continued to higher dimensions. However, the techniques we use have some formal similarities. We can construct $S$-matrix elements from the analytical nature of the amplitudes. However we fall a long way short of the exact $S$-matrices found in two dimensions. Nonetheless, we are able to construct enough of the $S$-matrix elements to determine large amounts of the ultraviolet structure of supergravity theories in dimension four or greater. In constructing amplitudes we attempt to use any and all information regarding the amplitude. Supersymmetry is one useful tool and in gravity theories with extended supersymmetry we can make more progress. For the maximal supergravity theory we can prove its non-renormalisability in $D=11$ and can conjecture the behavior in $D=4$.

\section{Technology}

Our philosophy is to evaluate the physical, onshell $S$-matrix from it's analytic properties. As far as possible we shall only consider on-shell objects. 


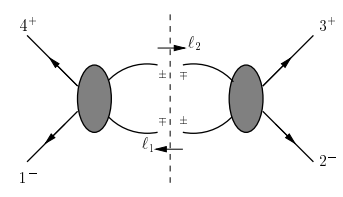

Figure 1:
A key property of the $S$-matrix is unitarity. Also within dimensional regularisation the amplitude is analytic in the dimension. The optical theorem, a con-

sequence of unitarity states

$$
2 \operatorname{Im} T=T^{\dagger} T
$$

In perturbation theory comparing both sides order by order relates, for example, the imaginary part of a one-loop amplitude to the product of tree amplitudes. In practical terms the imaginary part of a one loop amplitude is just the coefficient of a logarithm (or di- or polylogarithms) since,

$$
\ln \left(s_{i J}\right)=\ln \left(\left|s_{i j}\right|\right)+i \pi \Theta\left(s_{i j}\right)
$$

where $s_{i j}$ is one of the momentum invariants. Naively, the optical theorem only determines part of the one-loop amplitude since the amplitude may contain rational functions $f\left(s_{i j}\right)$ which have no imaginary part. However we can, by using dimensional regularisation, determine these rational parts also. Within dimensional regularisation the one-loop amplitude has a momentum weight of $-2 \epsilon\left(\right.$ since $\left.d^{D} p \longrightarrow d^{D-2 \epsilon}\right)$ This implies that the rational functions must be replaced by terms such as $f^{\prime}\left(s_{i j}\right)\left(s_{i j}\right)^{-2 \epsilon}$. Since

$$
\left(s_{i j}\right)^{-2 \epsilon}=1-2 \epsilon \ln \left(s_{i j}\right)
$$

the amplitude will pick up imaginary parts at $O(\epsilon)$. We thus deduce

knowledge of the cuts to all powers in $\epsilon$ will enable us to determine the amplitude

Of course this can be a painful computational burden in many circumstances. (Although in practice it is not always necessary to determine the cuts to all orders in $\epsilon$.) To see how this works consider the cut in a one-loop amplitude. Then the optical theorem states [i],

$$
\begin{aligned}
& \left.\operatorname{Disc} M^{1-l o o p}(1,2,3,4)\right|_{s-c u t}= \\
& i \int \sum_{\substack{\text { internal } \\
\text { particles }}} M^{\text {tree }}\left(-\ell_{1}^{s}, 1,2, \ell_{2}^{s^{\prime}}\right) M^{\text {tree }}\left(-\ell_{2}^{s^{\prime}}, 3,4, \ell_{1}^{s}\right)
\end{aligned}
$$

where the integral is over on-shell $\ell_{i}$. We must use this carefully within dimensional regularisation if we wish to determine the LHS correctly to all orders in $\epsilon$. The RHS contains tree amplitudes. Normally we do not regard these are depending upon $\epsilon$ however the momenta $\ell_{i}$ must match the loop momenta in the LHS. These are in $D-2 \epsilon$ dimensions so that the tree amplitudes should have the momenta $\ell_{i}$ in $D-2 \epsilon$ and the others in $D$ dimensions.

The analysis here is naturally merely indicative and the reader must be referred to elsewhere for the details of how this works and how it may be applied.

The optical theorem thus stated is a key ingredient to our calculational programme however it is not the only important input we also use some or all of the following features

- Amplitudes may in principle be calculated using Feynman diagrams. This allows us to restrict the "function" space an amplitude may lie in.

- Supersymmetric theories generally have simpler amplitudes which can be easier to calculate

- Field theory amplitudes may be calculated as the low energy limit of string theory amplitudes [i2i].

- Ámplitudes should have factorisation and collinear singularities when momentum invariants

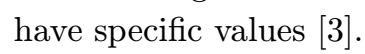

\section{3. $N=8$ Supergravity Amplitudes}

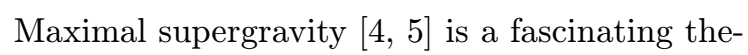
ory whose ultraviolet behavior is suspected but until the last few years has defied definite calculation. We shall attempt to determine this.

The one-loop amplitude was calculated many years ago by Green and Schwarz and Brink [6] to be

$\mathcal{M}_{4}^{\text {one-loop }}=\left(\frac{\kappa}{2}\right)^{2}$ stu $M_{4}^{\text {tree }}\left(\mathcal{I}_{4}(s, t)+2\right.$ perms. $)$

where $\mathcal{I}_{4}(s, t)$ is the $D$-dimensional scalar box integral (which may be easily evaluated). The one-loop amplitude is infinite in $D=8$ but not in other dimensions- on-shell. To determine the behavior in other dimensions we must go beyond a one-loop calculation. 
Using the technology described previously we have obtained a remarkably simple result for the final form for the two-loop four graviton ampli-

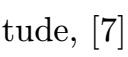

$$
\begin{aligned}
\mathcal{M}_{4}= & \left(\frac{\kappa}{2}\right)^{6} \text { stu } M_{4}^{\text {tree }}\left(s^{2} \mathcal{I}_{4}^{, \mathrm{P}}(s, t)+s^{2} \mathcal{I}_{4}^{, \mathrm{P}}(s, u)\right. \\
& \left.+s^{2} \mathcal{I}_{4}^{, \mathrm{NP}}(s, t)+s^{2} \mathcal{I}_{4}^{, \mathrm{NP}}(s, u)+\text { cyclic }\right) .
\end{aligned}
$$

where $\mathcal{I}_{4}^{\mathrm{P}}$ and $\mathcal{I}_{4}^{\mathrm{NP}}$ are two-loop scalar box integrals. They are the planar and non-planar box respectively. This amplitude has ultra-violet infinities in all dimension $D>6$. In particular there is a definite divergence in the maximal dimension $D=11$.

The two-loop ultraviolet divergences for $N=$ 8 supergravity in $D=7,9$ and 11 , are

$$
\begin{aligned}
& \left.\mathcal{M}_{4}^{D=7}\right|_{\text {pole }}=\frac{1}{2 \epsilon(4 \pi)^{7}} \frac{\pi}{3}\left(s^{2}+t^{2}+u^{2}\right) \times \mathcal{F}, \\
& \left.\mathcal{M}_{4}^{D=9}\right|_{\text {pole }}=\frac{1}{4 \epsilon(4 \pi)^{9}} \frac{-13 \pi}{9072}\left(s^{2}+t^{2}+u^{2}\right)^{2} \times \mathcal{F} \\
& \left.\mathcal{M}_{4}^{D=11}\right|_{\text {pole }}=\frac{1}{48 \epsilon(4 \pi)^{11}} \times \\
& \frac{\pi}{5791500}\left(438\left(s^{6}+t^{6}+u^{6}\right)-53 s^{2} t^{2} u^{2}\right) \times \mathcal{F}
\end{aligned}
$$

where $\mathcal{F}=(\kappa / 2)^{6} \times$ stu $M_{4}^{\text {tree }}$. There are no subdivergences because one-loop divergences are absent in odd dimensions when using dimensional regularisation. For even dimensions

$$
\begin{aligned}
&\left.\mathcal{M}_{4}^{D=8}\right|_{\text {pole }}=\frac{1}{2(4 \pi)^{8}} \times \\
&\left(-\frac{1}{24 \epsilon^{2}}+\frac{1}{144 \epsilon}\right)\left(s^{3}+t^{3}+u^{3}\right) \times \mathcal{F} \\
&\left.\mathcal{M}_{4}^{D=10}\right|_{\text {pole }}=\frac{1}{12 \epsilon(4 \pi)^{10}} \frac{-13}{25920} \times \\
& \text { stu }\left(s^{2}+t^{2}+u^{2}\right) \times \mathcal{F}
\end{aligned}
$$

The $1 / \epsilon^{2}$ pole in $D=8$ is precisely that need to cancel the $1 / \epsilon^{2}$ pole obtained when the oneloop counterterm is used used to calculate to two loops. The $1 / \epsilon$ pole shows how the expected nonpredictive nature of renormalisation occurs - new terms must be added to the Lagrangian order by order.

In all cases, for four graviton external states, the linearized counterterms take the form of derivatives acting on

$$
\begin{aligned}
t_{8} t_{8} & R^{4} \equiv t_{8}^{\mu_{1} \mu_{2} \cdots \mu_{8}} t_{8}^{\nu_{1} \nu_{2} \cdots \nu_{8}} R_{\mu_{1} \mu_{2} \nu_{1} \nu_{2}} \\
\times & R_{\mu_{3} \mu_{4} \nu_{3} \nu_{4}} R_{\mu_{5} \mu_{6} \nu_{5} \nu_{6}} R_{\mu_{7} \mu_{8} \nu_{7} \nu_{8}},
\end{aligned}
$$

plus the appropriate $N=8$ completion [8]" which also appears as the one-loop counterterm for $N=8$ supergravity in $D=8$. This particular tensor is well known from string theory am-

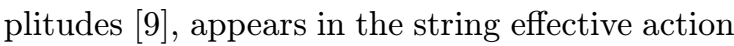
[10 id and is one of the higher dimensional analogs of the Bel-Robinson tensor [1] $\overline{1}_{1}^{\prime}$. It is consistent with $N=8$ supersymmetry which may not allow other possibilities.

For example the $D=11$ counterterm is a linear combination of the two tensors

$$
\begin{aligned}
& T^{A}=t_{8} t_{8} \cdot \partial_{\alpha \gamma \eta} R \partial^{\alpha \gamma \eta} R \partial_{\beta \delta \rho} R \partial^{\beta \delta \rho} R \\
& T^{B}=t_{8} t_{8} \cdot \partial_{\alpha \gamma \eta} R \partial^{\alpha \delta \eta} R \partial_{\beta \gamma \rho} R \partial^{\beta \delta \rho} R
\end{aligned}
$$

In each case the indices on the curvatures are contracted with the $t_{8}$ tensors and the indices on the derivative are contracted with each other. The $D=11$ counterterm is

$$
=-\frac{1}{48 \epsilon(4 \pi)^{11}} \times \frac{\pi}{5791500}\left(\frac{2575}{12} T^{A}+\frac{53}{6} T^{B}\right)
$$

\section{Higher-loop conjecture}

To determine the behavior for $D \leq 6$ we need to go beyond two loops. As yet this remains a very challenging calculation. In order to specify the precise form of the conjecture at $L$ loops one would need to investigate cuts with up to $(L+1)$ intermediate particles. Nevertheless, some of the integral coefficients and numerators can be obtained from the two-particle cuts. If we assume these pieces of the amplitude are representation we can conjecture the ultra-violet structure. Of course in the absence of definite calculations this remains very much a guess - however a guess which we expect will prove correct.

By examine some of the cuts we can identify the most divergent pieces as

$$
\int\left(d^{D} p\right)^{L} \frac{\left(p^{2}\right)^{2(L-2)}}{\left(p^{2}\right)^{3 L+1}} .
$$

This integral will be finite when

$$
D<\frac{10}{L}+2, \quad(L>1)
$$

The results of this analysis are summarized in table 1 . In particular, in $D=4$ no threeloop divergence appears - contrary to expectations from a superspace analysis $[12,1,13$ 
first $R^{4}$-type counterterm occurs at five loops. The divergence will have the same kinematical structure as the $D=7$ two-loop divergence, but with a different non-vanishing numerical coefficient.

\begin{tabular}{|c|c|l|c|}
\hline Dimension & Loop & Degree & Counterterm \\
\hline 8 & 1 & log. & $R^{4}$ \\
\hline 7 & 2 & log. & $\partial^{4} R^{4}$ \\
\hline 6 & 3 & quad. & $\partial^{6} R^{4}$ \\
\hline 5 & 4 & quad. & $\partial^{6} R^{4}$ \\
\hline 4 & 5 & log. & $\partial^{4} R^{4}$ \\
\hline
\end{tabular}

Table 1: The relationship between dimensionality and the number of loops at which the first ultraviolet divergence should occur in the $N=8$ supergravity four-point amplitude. The form of the associated counterterm assumes the use of dimensional regularisation.

\section{Non-maximal Supergravity}

It is interesting to compare the structures found between types II and type I supergravity (and their lower dimensional descendants.) We have examined the one-loop structures for dimensions $4 \leq D \leq 10\left[1 \overline{1}_{1}\right]$ however here we shall restrict presentation to the features of the $D=8$ case.

In $D=8$ power counting indicates that the counterterms will be of the form $R^{4}$. There are seven independent $R^{4}$ tensors [1] $\left.{ }_{1}^{1}\right]$ (in $D<8$ these are no longer independent.) ${ }^{1}$

$$
\begin{aligned}
& T_{1}=\left(R_{p, q, r, s} R_{p, q, r, s}\right)^{2} \\
& T_{2}=\left(R_{p, q, r, s} R_{p, q, r, t}\right)\left(R_{p^{\prime}, q^{\prime}, r^{\prime}, s} R_{p^{\prime}, q^{\prime}, r^{\prime}, t}\right) \\
& T_{3}=R_{p, q, r, s} R_{p, q, t, u} R_{t, u, v, w} R_{r, s, v, w} \\
& T_{4}=R_{p, q, r, s} R_{p, q, t, u} R_{r, t, v, w} R_{s, u, v, w} \\
& T_{5}=R_{p, q, r, s} R_{p, q, t, u} R_{r, v, t, w} R_{s, v, u, w} \\
& T_{6}=R_{p, q, r, s} R_{p, t, r, u} R_{t, v, u, w} R_{q, v, s, w} \\
& T_{7}=R_{p, q, r, s} R_{p, t, r, u} R_{t, v, q, w} R_{u, v, s, w}
\end{aligned}
$$

On shell the combination

$$
-\frac{T_{1}}{16}+T_{2}-\frac{T_{3}}{8}-T_{4}+2 T_{5}-T_{6}+2 T_{7}
$$

${ }^{1}$ The Riemann tensor is undistinguished from the Weyl tensor in our $R^{4}$ terms for on-shell four point amplitudes. vanishes (or rather is a total divergence) being proportional to the Euler form.

In order to calculate the appropriate $N=8$ counterterm we evaluate the (on-shell) amplitude and we find it factorises in the following way:

$$
M^{N=8, D=8}=\frac{1}{\epsilon} \times\left(\frac{\kappa}{2}\right)^{4} \frac{i}{(4 \pi)^{4}} \frac{1}{2} K_{1} \times K_{1}
$$

where

$$
\begin{aligned}
K_{1} & =t u\left(\epsilon_{1} \cdot \epsilon_{2}\right)\left(\epsilon_{3} \cdot \epsilon_{4}\right) \\
& +2\left(\epsilon_{1} \cdot \epsilon_{2}\right)\left(t\left(\epsilon_{3} \cdot k_{1} \epsilon_{4} \cdot k_{2}\right)+u\left(\epsilon_{3} \cdot k_{2} \epsilon_{4} \cdot k_{1}\right)\right) \\
& + \text { cyclic terms }
\end{aligned}
$$

The counterterm necessary to cancel this infinity is,

$\frac{1}{\epsilon}\left(\frac{\kappa}{2}\right)^{4} \frac{i}{(4 \pi)^{4}} \frac{1}{4}\left[-\frac{T_{1}}{16}+T_{2}-\frac{T_{3}}{8}-0 . T_{4}+2 T_{5}-T_{6}-2 T_{7}\right]$

The other case is $N=4$ supergravity. By this we mean the type I supergravity in $\mathrm{D}=10$ and its dimensional descendants. There is of course both a matter multiplet and a gravity multiplet (which contains the graviton.) The $N=8$ multiple is a sum of these so only one is independent from the $N=8$ case. For the graviton amplitude with states in the matter multiplet circulating in the loop the infinity is

$$
M^{N=4, D=8}=\frac{1}{\epsilon} \times\left(\frac{\kappa}{2}\right)^{4} \frac{i}{(4 \pi)^{4}} \times \frac{1}{720} K_{1} \times K_{2}
$$

where

$$
\begin{aligned}
& K_{2}=-\epsilon_{1} \cdot \epsilon_{2} \epsilon_{3} \cdot \epsilon_{4}\left(3 t^{2}+5 t u+3 u^{2}\right)+\cdots \\
& +2 \epsilon_{1} \cdot \epsilon_{2}\left(3 s \epsilon_{3} \cdot k_{4} \epsilon_{4} \cdot k_{3}+t \epsilon_{3} \cdot k_{1} \epsilon_{4} \cdot k_{2}+u \epsilon_{3} \cdot k_{2} \epsilon_{4} \cdot k_{1}\right)+\cdots \\
& -12\left(k_{2} \cdot \epsilon_{1} k_{1} \cdot \epsilon_{2} k_{4} \cdot \epsilon_{3} k_{3} \cdot \epsilon_{4}+k_{3} \cdot \epsilon_{1} k_{4} \cdot \epsilon_{2} k_{1} \cdot \epsilon_{3} k_{2} \cdot \epsilon_{4}\right. \\
& \left.\quad+k_{4} \cdot \epsilon_{1} k_{3} \cdot \epsilon_{2} k_{2} \cdot \epsilon_{3} k_{1} \cdot \epsilon_{4}\right)
\end{aligned}
$$

where $+\cdots$ indicates the necessary terms we must add. We have organised $K_{2}$ according to the number of $\epsilon_{i} \cdot \epsilon_{j}$. The counterterms necessary to cancel this are

$$
\begin{aligned}
-\frac{1}{\epsilon}\left(\frac{\kappa}{2}\right)^{4} \frac{i}{(4 \pi)^{4}} \frac{1}{11520}\left(-3 T_{1}\right. & +24 T_{2}-6 T_{3}+4 T_{4} \\
& \left.+0 . T_{5}+0 . T_{6}+32 T_{7}\right)
\end{aligned}
$$

We can relate this also to specific tensors contracted against $R^{4}$. The tensor $t_{8}$ can be split 
into two pieces $t_{(12)}$ and $t_{(48)} t_{8}=\frac{1}{2}\left(t_{(12)}+\right.$ $\left.t_{(48)}\right)$ each having the same symmetry properties as $t_{8}$. The tensors $t_{(12)}$ and $t_{(48)}$ contain 12 and 48 quartic monomials in the $\delta$ 's respectively and are the only two tensors which have the same symmetry properties of $t_{8}$ in eight dimensions [ịn].

$$
\begin{aligned}
& t_{(12)}^{i j k l m n p q}=-\left(\left(\delta^{i k} \delta^{j l}-\delta^{i l} \delta^{j k}\right)\left(\delta^{m p} \delta^{n q}-\delta^{m q} \delta^{n p}\right)\right. \\
&+\left(\delta^{k m} \delta^{l n}-\delta^{k n} \delta^{l m}\right)\left(\delta^{p i} \delta^{q j}-\delta^{p j} \delta^{q i}\right) \\
&\left.+\left(\delta^{i m} \delta^{j n}-\delta^{i n} \delta^{j m}\right)\left(\delta^{k p} \delta^{l q}-\delta^{k q} \delta^{l p}\right)\right) \\
& t_{(48)}^{i j k l m n p q}=\left(\delta^{j k} \delta^{l m} \delta^{n p} \delta^{q i}+\delta^{j m} \delta^{n k} \delta^{l p} \delta^{q i}\right. \\
&\left.+\delta^{j m} \delta^{n p} \delta^{q k} \delta^{l i}+[i \leftrightarrow j]+[k \leftrightarrow l]+[m \leftrightarrow n]\right)
\end{aligned}
$$

where $[i \leftrightarrow j]$ denotes antisymmetrisation with respect to $i$ and $j$. From these tensors we can define

$$
\begin{gathered}
A=\frac{1}{4} t_{(12)} t_{(12)} \cdot R^{4}, \quad B=\frac{1}{4} t_{(12)} t_{(48)} \cdot R^{4} \\
C=\frac{1}{4} t_{(48)} t_{(48)} \cdot R^{4}
\end{gathered}
$$

where the - denotes the usual contraction of the upper and lower eight indices.

( We can also express these tensor contractions as traces $\left[\overline{1}_{1}^{1}\right.$.). $t_{8} t_{(12)} \cdot R^{4}=48 t_{8} \operatorname{Tr}\left(R^{4}\right)$ etc )

In terms of these combinations the $N=8$ counterterm of the type $t_{8} t_{8} R^{4}$ is just

$$
\frac{1}{768}(A+2 B+C)
$$

and the $N=4$ matter contributions is proportional to $(2 A+C)$.

We have obtained very similar results for $D=$ 10. In $D=10$ the $N=8$ supergravity amplitude vanishes (onshell) but the two $N=4$ components do not. For these the counterterm also factorises in the form $\sim K_{1} \times L_{i}$ where the tensors $L_{i}$ contain two more powers of momenta than the $K_{i}$.

\section{Relationships between gravity and Yang-Mills}

Analysing the structure in amplitudes can reveal strong parallels between gravity and Yang-Mills calculation. In many ways the gravity results appear as the "square" Yang-Mills. In fact, calculations in $N=4$ Yang-Mills have been used

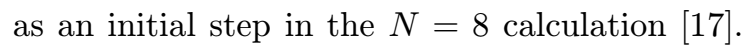
To extension to $N=8$ involved to some extend repeating the calculation whilst squaring the algebra.

Relationships between the tree amplitudes of Yang-Mills were obtained from the low energy limit of string theory by Kawai Lewellen and Tye [1] $\overline{8}]$. They found a series of algebraic relationships between the two sets of tree amplitudes. At four point

$M_{4}^{\text {tree }}(1,2,3,4)=-i s_{12} A_{4}^{\text {tree }}(1,2,3,4) A_{4}^{\text {tree }}(1,2,4,3)$,

where $M_{4}$ is the gravity amplitude and $A_{4}$ is the Yang-Mills colour ordered tree amplitude. The KLT relationships have proved extremely useful, however the trees can also be rearranged as in fig. 2 where we have rearranged the amplitudes to be in the form

\section{kinematic polynomial $\times$ pole structure}

This can be done so that the relatiobship between $\left.{ }^{C_{s}}{ }_{1}^{2}\right\rangle\left\langle\left\langle_{4}^{3}\right.\right.$ gravity and YangMills is clear [i] $\left.\overline{1}_{1}^{\prime}\right]$ we keep the pole structure and square the multiplying polynomial. Note that Figure 2: The tree amplithere is some free- tudes can be arranged to disdom in this since play a simple squaring relationwe can more terms ship between the Yang-Mills between the dif- and Gravity Cases ferent coefficients to some extent.

When we consider the one-loop amplitudes we find a very similar relationship. The amplitude can be written as a coefficient times an integral function. For the case of $N=8$ supergravity the comparison to the $N=4$ super Yang-Mills is shown in fig. 3 As we can see again we find that the coefficient of the integral is squared. 
For the two loop case we find the situation as shown in fig.4. (Overall factors of $s t A^{\text {tree }}$ and $\left[s t A^{\text {tree }}\right]^{2}$ have been ommitted for clarity.) Whilst calculating the supergravity amplitude this relationship was postulated which allowed

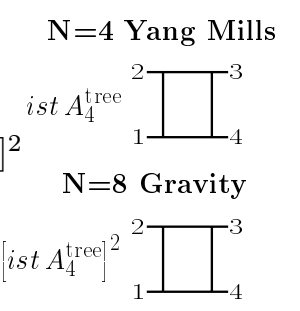

Figure 3: an anasatz for the supergravity amplitude to be quickly made. With a specific ansatz checking the cuts was relatively straightforward.

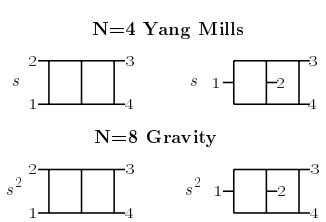

Figure 4: A simple relationship between the amplitudes of maximal SUSY and SUGRA persists to two loops

\section{References}

[1] L.D. Landau, Nucl. Phys. B13:181 (1959); S. Mandelstam, Phys. Rev. 112:1344 (1958); Phys. Rev. 115:1741 (1959); R.E. Cutkosky, J. Math. Phys. 1:429 (1960); Z. Bern, L. Dixon, D.C. Dunbar and D.A. Kosower, Nucl. Phys. B425:217 (1994) hep-ph/9403226; Nucl. Phys. B435:59 (1995) hep-ph/9409265.

[2] Z. Bern and D.A. Kosower, Nucl. Phys. B379:451 (1992); Z. Bern, D.C. Dunbar and T. Shimada, Phys. Lett. B312:277 (1993) hep-th/9307001;; D.C. Dunbar and P.S. Norridge, Nucl. Phys. B433:181 (1995) hep-th/9408014; Z. Bern and D.C. Dunbar, Nucl. Phys. B379:562 (1992) Z. Bern, Phys. Lett. B296:85 (1992);

[3] Z. Bern and A.G. Morgan, Nucl. Phys. B467:479 (1996) hep-ph/9511336; Z. Bern, G. Chalmers, L. Dixon and D.A. Kosower, Phys. Rev. Lett. 72:2134 (1994) hep-ph/9312333;

Z. Bern and G. Chalmers, Nucl. Phys. B447:465 (1995) hep-ph/9503236!

[4] E. Cremmer and B. Julia, Phys. Lett. B80:48 (1978)); Nucl. Phys. B159:141 (1979).
[5] E. Cremmer, B. Julia and J. Scherk, Phys. Lett. B76:409 (1978);

[6] M.B. Green, J.H. Schwarz and L. Brink, Nucl. Phys. B198:474 (1982);

[7] Z. Bern, L. Dixon, D.C. Dunbar, M. Perelstein and J.S. Rozowsky, Nucl. Phys. B530:401 (1998) hep-th/9802162.

[8] S. Deser and D. Seminara, Phys. Rev. Lett. 82:2435 (1999), hep-th/9812136.

[9] M.B. Green, J.H. Schwarz and E. Witten, Superstring Theory (Cambridge University Press, 1987);

[10] M.B. Green, M. Gutperle and P. Vanhove, Phys. Lett. B409:177 (1997), 'hep-th/9706175; J.G. Russo and A.A. Tseytlin, Nucl. Phys. B508:245 (1997), hep-th/9707134!

[11] I. Robinson, unpublished;

L. Bel, Acad. Sci. Paris, Comptes Rend. 247:1094 (1958) and 248:1297 (1959).

[12] P.S. Howe, K.S. Stelle and P.K. Townsend, Nucl. Phys. B236:125 (1984).

[13] P.S. Howe and K.S. Stelle, Phys. Lett. B137:175 (1984) Int. J. Mod. Phys. A 4:1871 (1989).

[14] D.C. Dunbar, B. Julia, D. Seminara and M. Trigiante, J. High Ener. Phys. 0001:046 (2000) hep-th/9911158.

[15] S.A. Fulling, R.C. King, B.G. Wybourne and C.J. Cummins Class. and Quant. Grav. 9:1151 (1992).

[16] A. Tseytlin, Nucl. Phys. B467:383 (1996);

[17] Z. Bern, J.S. Rozowsky and B. Yan, Phys. Lett. B401:273 (1997) hep-ph/9702424!.

[18] H. Kawai, D.C. Lewellen and S.-H.H. Tye, Nucl. Phys. B269:1 (1986).

[19] Z. Bern and A.K. Grant, Phys. Lett. B457:23 (1999), hep-th/9904026

[20] D.C. Dunbar and P.S. Norridge, Class. and Quant. Grav. 14:351 (1997) hep-th/9512084 P.S. Norridge, Phys. Lett. B387:701 (1996) hep-th/9606067. 